\title{
Fatty liver deposition and sparing: a pictorial review
}

\author{
Patrick-Olivier Décarie • Luigi Lepanto • Jean-Sébastien Billiard • Damien Olivié • \\ Jessica Murphy-Lavallée • Claude Kauffmann • An Tang
}

Received: 8 February 2011 /Revised: 17 March 2011 / Accepted: 9 June 2011 / Published online: 28 June 2011

(C) European Society of Radiology 2011

\begin{abstract}
Objective Fatty liver deposition is a very common finding, but it has many atypical patterns of distribution that can represent diagnostic pitfalls. The purpose of this pictorial essay is to review different patterns of fatty liver deposition and sparing. Methods We searched our archive retrospectively, reviewed the literature, and identified six patterns of liver steatosis. Results Steatosis may be diffuse, geographic, focal, subcapsular, multifocal or perivascular.

Conclusions Previous knowledge of atypical patterns of steatosis distribution may prevent misdiagnosis of infiltrative disease or focal liver lesions. When an unusual form of fatty liver deposition is suspected on ultrasound or computed tomography, magnetic resonance imaging may be used to confirm the diagnosis.
\end{abstract}

Keywords Fatty liver deposition · Fatty liver sparing · Chemical shift gradient-echo $\cdot \mathrm{CT} \cdot \mathrm{MRI}$

\section{Introduction}

Fatty liver disease is a very common condition, with a prevalence of $20-30 \%$ in the adult population [1] and $70 \%$

P.-O. Décarie $\cdot$ L. Lepanto $\cdot$ J.-S. Billiard $\cdot$ D. Olivié $\cdot$

J. Murphy-Lavallée $\cdot$ A. Tang $(\bowtie)$

Department of Medical Imaging, University of Montreal and

Centre de Recherche du Centre Hospitalier de l'Université de

Montréal (CRCHUM), Hôpital Saint-Luc,

1058 rue Saint-Denis,

Montréal, QC, Canada H2X 3J4

e-mail: duotango@gmail.com

C. Kauffmann

Department of Medical Imaging,

University of Montreal and CRCHUM, Hôpital Notre-Dame,

1560 rue Sherbrooke Est,

Montréal, QC, Canada H2L 4M1 in diabetes patients [2]. Among patients who develop nonalcoholic steatohepatitis (NASH), half will evolve to fibrosis, $10-15 \%$ to cirrhosis and $5.4 \%$ to hepatic insufficiency [1]. Radiologists must report fatty liver disease because it may have serious long-term consequences if left untreated. Recent trials have shown histological improvements of steatosis and NASH after a medical treatment [3].

Fatty liver may present with different patterns of deposition and sparing. We searched our archive retrospectively, reviewed the literature and identified six patterns of liver steatosis: diffuse, geographic, focal, subcapsular, multifocal and perivascular. Diffuse forms may be graded subjectively according to severity (mild, moderate, severe) or quantitatively with magnetic resonance (MR)-based methods [4]. Some unusual focal forms may be misdiagnosed as infiltrative or nodular liver lesions. Therefore, previous knowledge of their cross-sectional appearance may prevent unwarranted investigations or allow non-invasive diagnosis.

Ultrasound is the first-line imaging technique for the screening and follow-up of patients with chronic liver disease. It is, therefore, important for radiologists to be aware of patterns of heterogeneous or focal forms of fatty deposition. On ultrasound, fatty liver is hyperechoic compared with renal cortex and spleen, whereas fatty sparing is isoechoic or hypoechoic.

On unenhanced computed tomography (CT), liver density less than 40 Hounsfield units (HU) [5] or a density difference of more than $10 \mathrm{HU}$ between spleen and liver indicates fatty liver [6]. Alternatively, liver parenchyma hypodensity relative to vessels establishes the presence of moderate to severe fatty liver disease [7]. On portal phase contrast-enhanced CT with a standardised protocol, a density difference greater than $25 \mathrm{HU}$ between spleen and liver suggests fatty liver deposition [6]. CT with contrast material was previously thought to be less reliable in detecting fatty liver. However, recent data suggest that 
Fig. 1 a-d Severe diffuse fatty liver disease. a Ultrasound image in a 58-year-old man known for intraductal papillary mucinous neoplasm (IPMN/IPMT) of the pancreas and alcoholic pancreatitis showing a hyperechoic liver compared with the right renal cortex. b CT, axial contrastenhanced portal phase image shows a diffusely hypodense liver (42 HU) compared with the spleen (113 HU) (>25 HU difference). MRI, c axial T1weighted in-phase and d out-ofphase images show an important signal drop of the liver on the opposed-phase image. MR images were obtained on a 1.5 -T MRI system with TEs of 2.2 and 4.5 for in- and out-of-phase images respectively
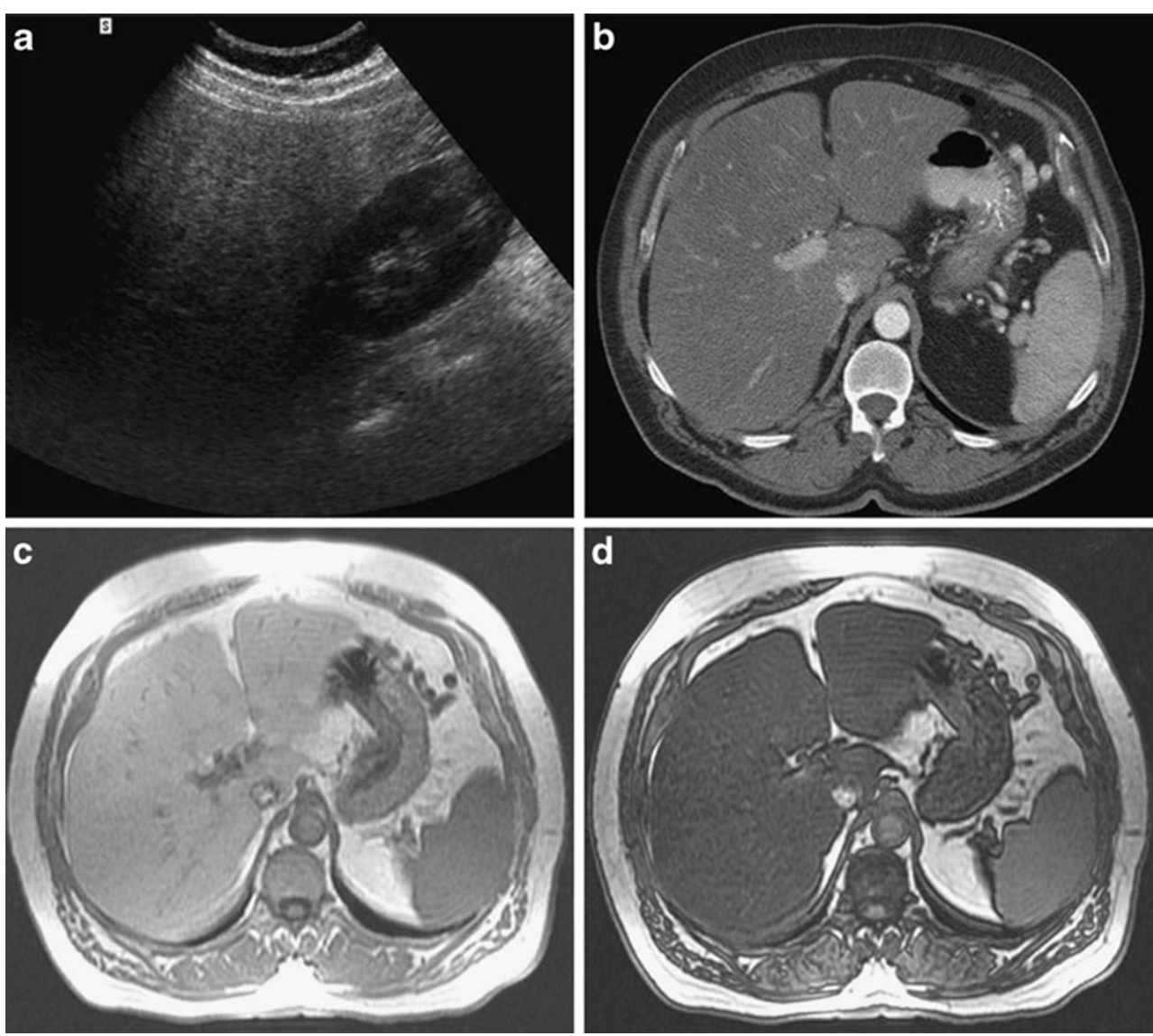

portal phase contrast-enhanced CT using blood-subtracted hepatic attenuation may have a similar accuracy to or even greater accuracy than unenhanced CT in the diagnosis of fatty liver [8].

MR imaging (MRI) allows measurement of fat and water proton signals and is considered the most accurate noninvasive technique for hepatic fat quantification [4]. Chemical shift gradient-echo imaging is a widely available MRI technique for fat detection and quantification. Fatty liver shows a signal drop on gradient echo T1-weighted out-of-phase images compared with in-phase images [7]. The dual-echo method results in underestimation of the fat fraction by only $2.9 \%$ compared with spectroscopy, the non-invasive "gold standard" [9].

Diffuse fat deposition

Diffuse fatty liver deposition is the most prevalent form of fatty liver disease. The liver is homogeneously involved by fat accumulation (Fig. 1). It may have different levels of severity that can be determined by using quantification maps. The presence of diffuse steatosis in a patient with hepatomegaly and an enlarged caudate-to-right lobe ratio should raise the possibility of NASH [10].
Fig. 2 a, bGeographic fat accumulation limited to the right lobe of the liver. MRI of the liver in a 57-year-old woman known for multiple focal nodular hyperplasia. a Axial T1weighted in-phase and $\mathbf{b}$ out-ofphase images show an important signal drop in the right lobe of the liver on opposed-phase image
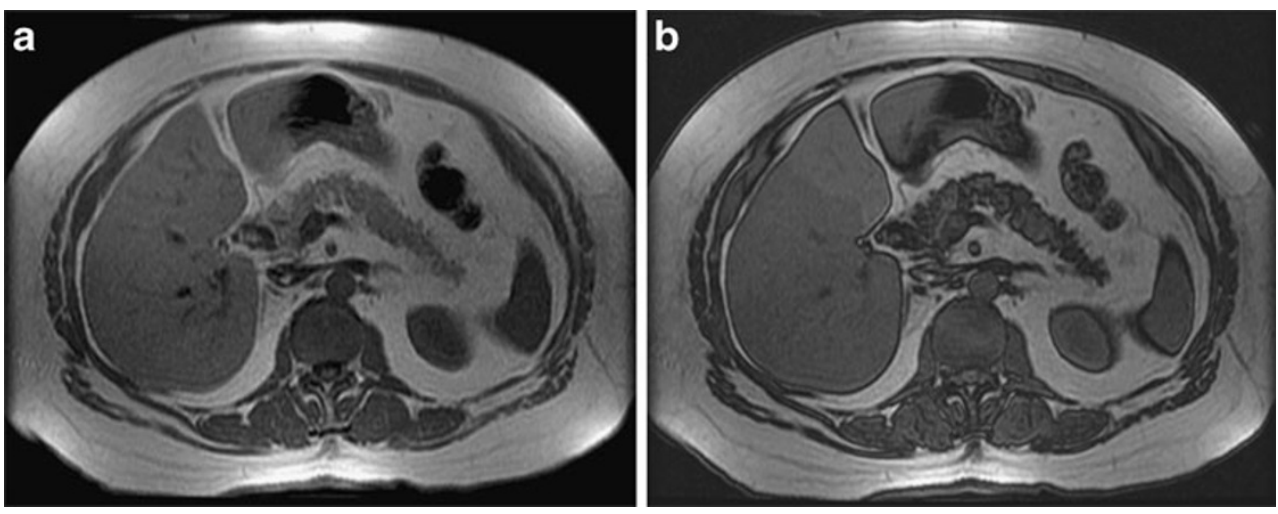
Fig. 3 a-c Severe geographic fatty liver deposition. MRI of the liver in a 76-year-old man who had hepatico-jejunostomy for biliary duct trauma and stenosis followed by two episodes of cholangitis in 1997 and 2006. a Axial T1-weighted inphase and $\mathbf{b}$ out-of-phase images show an important signal drop on the opposed-phase image in a geographic distribution, affecting the hepatic dome more severely. c Subtraction confirms severe geographic fatty liver deposition

Fig. 4 Focal fatty liver deposition. a CT, axial contrastenhanced portal phase image in a 58-year-old woman shows a hypodense, mildly heterogeneous, cuneiform lesion in the posterior aspect of segment IV. MRI, b axial T1-weighted inphase and $\mathbf{c}$ out-of-phase images show a signal drop within this lesion on the opposed-phase image
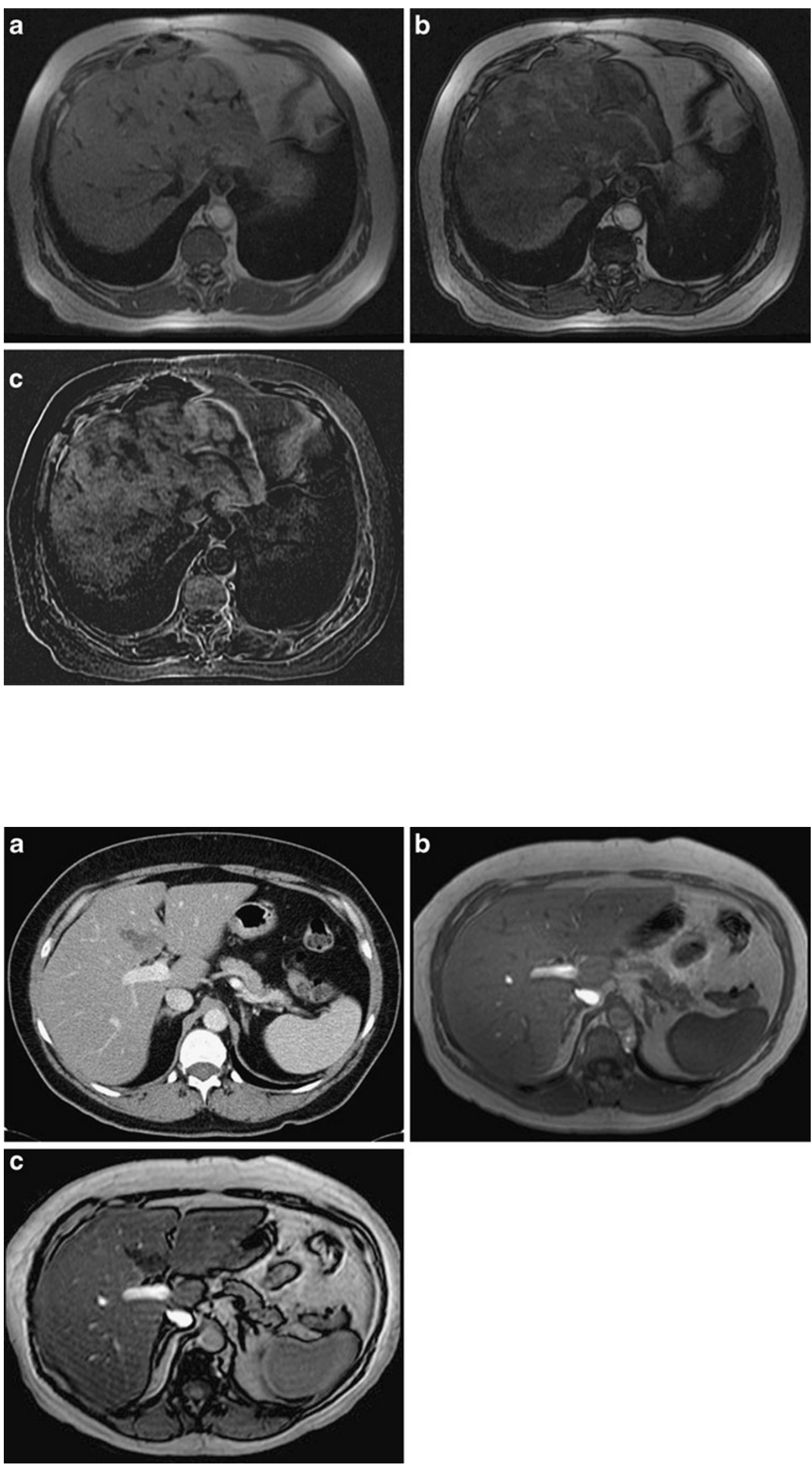
Fig. 5 a-d Perivesicular fatty sparing. a Ultrasound image in a 77-year-old woman who had nephrectomy for renal cell carcinoma shows a hyperechoic liver with hypoechoic perivesicular foci. b CT, axial contrastenhanced portal phase image shows a diffusely hypodense liver $(57 \mathrm{HU})$ in comparison with the spleen $(135 \mathrm{HU})$ with a perivesicular spared zone (in segments IV and V). MRI, c axial T1-weighted in-phase and d out-of-phase images show an important signal drop of the liver on the opposed-phase image with the exception of a perivesicular spared zone
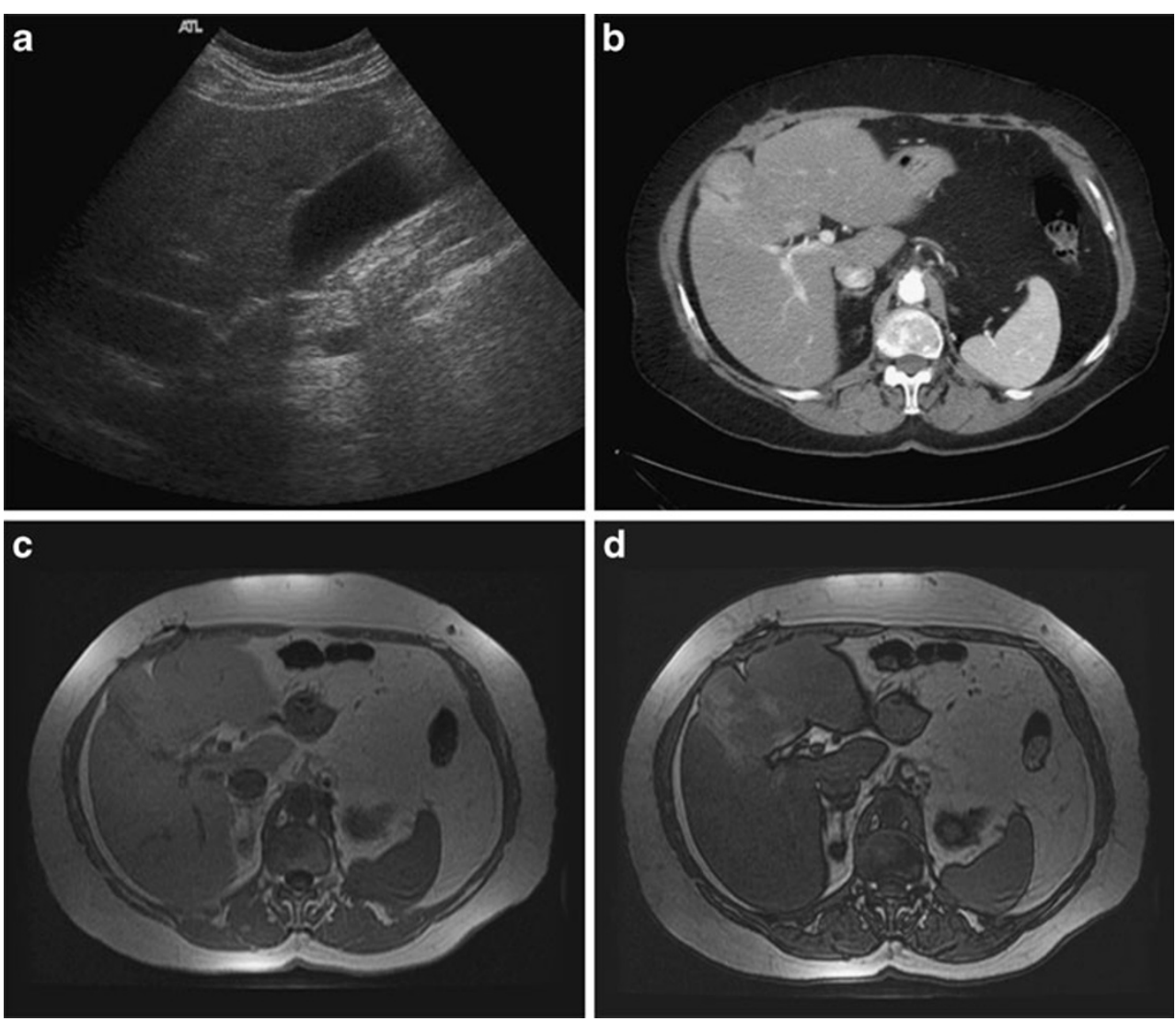

Geographic fat deposition

Geographic fatty liver disease is a frequently encountered variant. Different geographic patterns can be attributed to specific causes. For example, fat accumulation sometimes occurs only in the right lobe (Fig. 2). One hypothesis to explain this distribution is that blood from the superior mesenteric vein containing lipogenic alimentary factors is preferentially distributed to the right lobe of the liver [11]. Geographic patterns may be secondary to an insult to the liver parenchyma. For example, fatty liver deposition can be distributed in territories previously affected by cholangitis (Fig. 3).

Focal fat deposition and focal fatty sparing

Focal fat deposition is slightly less common and can mimic other hepatic benign or malignant lesions on ultrasound and CT (Fig. 4). MRI is very useful for making the diagnosis of focal hepatic steatosis, which appears isointense or hyperintense to liver on in-phase images and loses signal on outof-phase images. Fat accumulation does not show diffusion
Fig. 6 a-c Subcapsular fatty liver deposition. a Axial contrast-enhanced portal phase CT image in a 58-year-old man with a neuro-endocrine tumour of the pancreas shows two small hypodense subcapsular hepatic lesions in segment V. MRI, b axial T1-weighted in-phase and c out-of-phase images show a drop of signal within the two subcapsular lesions on the opposed-phase image
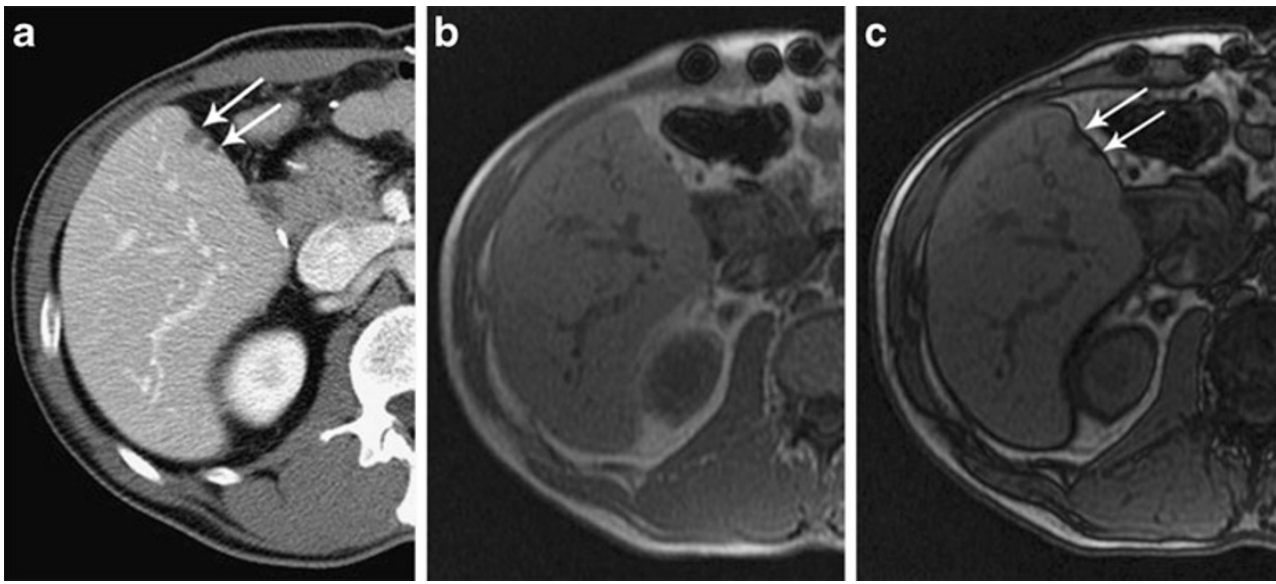
Fig. 7 a-d Multifocal fatty liver deposition. a Ultrasound image in a 39-year-old woman with lymphoma and malignant thymoma with local pleural recurrence treated with radiotherapy and chemotherapy shows multiple nodular hyperechoic foci throughout the liver. b CT, axial unenhanced image shows multiple hypodense foci throughout the liver. MRI, $\mathbf{c}$ axial T1-weighted in-phase and d outof-phase images show multiple lesions with signal drop on the opposed-phase image
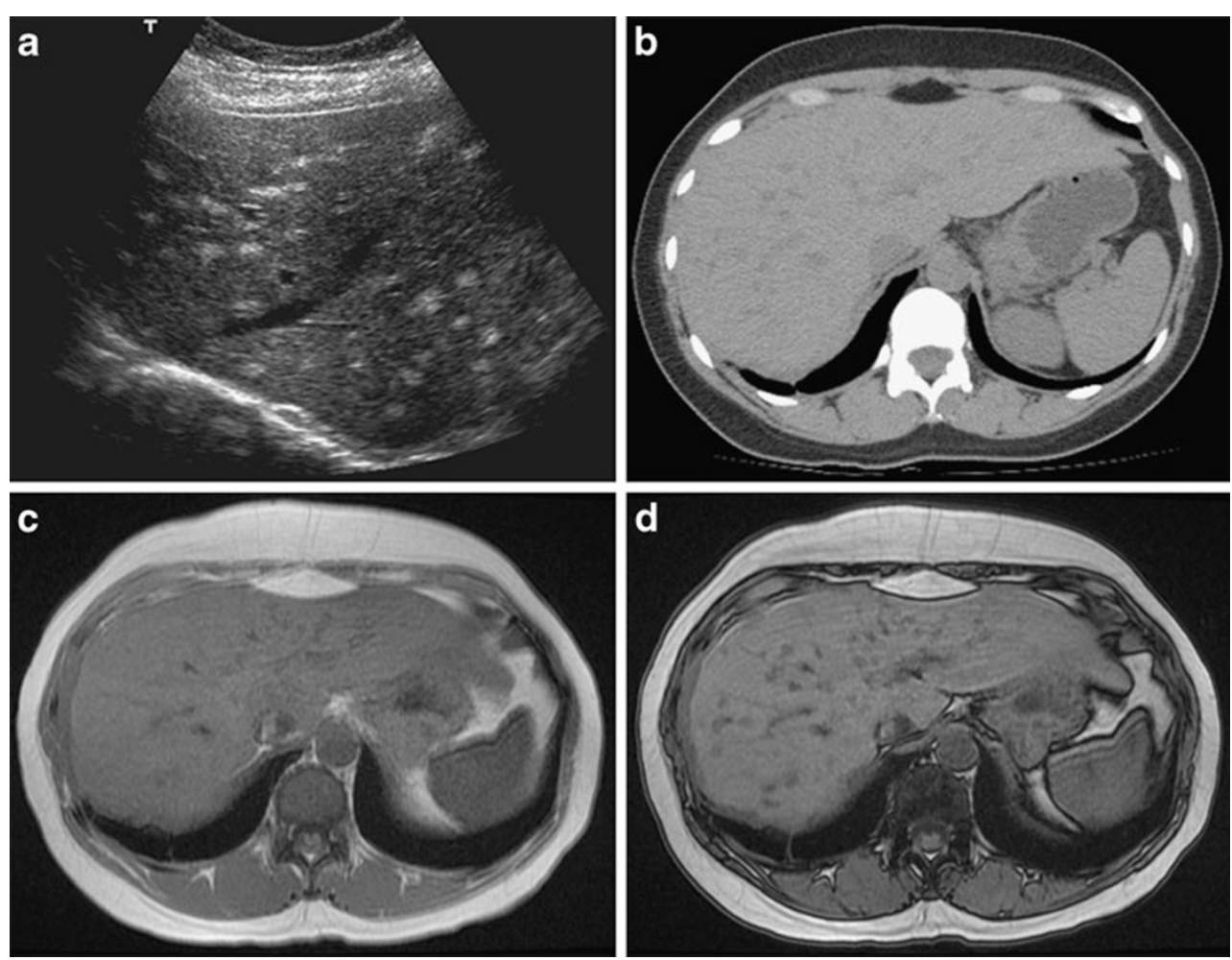

restriction, which may also be useful to differentiate steatosis from other liver abnormalities. Moreover, focal fat accumulation tends to show wedge-shaped margins, no mass effect on adjacent blood vessels or the biliary tree, sharp boundaries, and lobar or segmental distribution [12].

Some regions of the liver are more prone to being affected by focal fat deposition. The same regions correspond to spared areas in diffuse fat deposition (Fig. 5). Those are the perivesicular and subcapsular regions abutting the hepatic hilum or falciform ligament in the area drained by the Sapey's vein [7, 11]. The classical patterns of focal fatty liver deposition and sparing are linked to vascular anatomical variants that involve capsular veins, peribiliary veins, the cystic vein (which drains perivesicular areas), and the right gastric vein (which drains the anterior portion of segment I and the posterior portion of segment IV). These veins can be the source of an aberrant venous draining system without portal inflow causing local haemodynamic anomalies that may lead to focal fatty liver deposition and sparing $[11,13]$.

Subcapsular fat deposition

Subcapsular fat deposition may present as small fat nodules (Fig. 6) or as a confluent peripheral region of fat confined to a subcapsular zone [7]. It may be caused by insulin added to the peritoneal dialysate in certain patients with renal failure and insulin-dependent diabetes. Subcapsular
Fig. 8 a, b Perivascular fatty sparing. a Ultrasound image in a 39-year-old man with a clinical suspicion of non-alcoholic fatty liver disease based on elevated liver enzymes shows a geographic area of fatty sparing parallel to the right hepatic vein. b Doppler image shows an absence of mass effect on the right hepatic vein
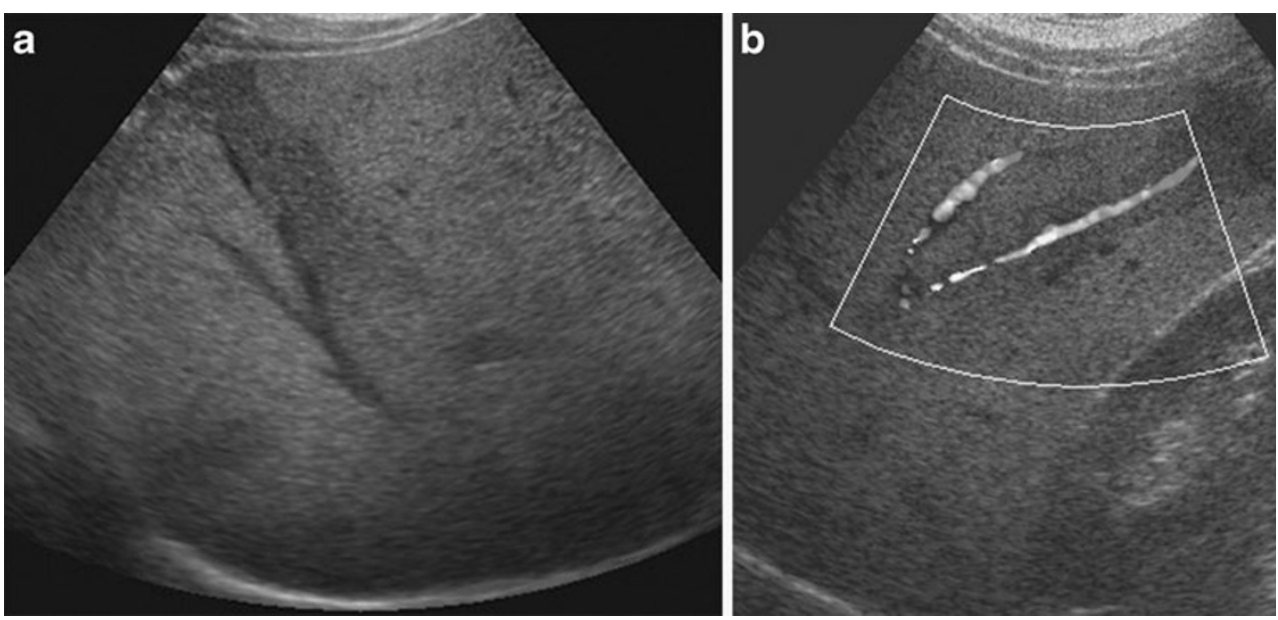
hepatocytes are exposed to a higher concentration of insulin than the rest of the liver in those patients. Because insulin promotes the esterification of free fatty acids into triglycerides, the peritoneal administration of insulin can result in subcapsular fat accumulation [14]. This form of fatty liver deposition may also be idiopathic.

\section{Multifocal fat deposition}

Multifocal fatty liver deposition is another unusual pattern that can represent a difficult diagnostic challenge. This entity has also been described as multinodular hepatic steatosis [15]. Multiple fat foci are randomly distributed throughout the liver. The foci are usually round or oval and may mimic multiple nodules. The differential diagnosis on $\mathrm{CT}$ and ultrasound may include metastasis, lymphoma, sarcoidosis, abscesses, candidiasis, haemangiomatosis and biliary hamartomas. MRI is usually required to make the diagnosis (Fig. 7). The pathogenesis of multifocal fat deposition is unknown.

\section{Perivascular fat deposition and perivascular fatty sparing}

A very unusual pattern of fatty liver disease is perivascular deposition. Halos of fat surround the hepatic veins, the portal veins, or both hepatic and portal veins [7]. Perivascular fatty sparing can also be seen (Fig. 8). It may be difficult to differentiate perivascular fat deposition or perivascular sparing from periportal oedema, passive hepatic congestion and Budd-Chiari syndrome on CT and ultrasound. MRI is useful for making this rare diagnosis by showing a signal intensity loss on opposed-phase images compared with in-phase images. The pathogenesis of perivascular fat deposition and sparing is unknown.

\section{Conclusion}

Fatty liver deposition is a very common finding but it has many atypical patterns of distribution that can represent diagnostic pitfalls. Steatosis may be diffuse, geographic, focal, subcapsular, multifocal or perivascular. When an atypical form of fatty liver deposition is suspected on ultrasound or CT, MRI may be used to confirm the diagnosis because of its ability to break down fat and water signal components.

\section{References}

1. Preiss D, Sattar N (2008) Non-alcoholic fatty liver disease: an overview of prevalence, diagnosis, pathogenesis and treatment considerations. Clin Sci (Lond) 115(5):141-150

2. Targher G, Bertolini L, Padovani R, Rodella S, Tessari R, Zenari L et al (2007) Prevalence of nonalcoholic fatty liver disease and its association with cardiovascular disease among type 2 diabetic patients. Diabetes Care 30(5):1212-1218

3. Sanyal AJ, Chalasani N, Kowdley KV, McCullough A, Diehl AM, Bass NM et al (2010) Pioglitazone, Vitamin E, or placebo for nonalcoholic steatohepatitis. N Engl J Med 362(18):1675-1685

4. Cassidy FH, Yokoo T, Aganovic L, Hanna RF, Bydder M, Middleton MS et al (2009) Fatty liver disease: MR imaging techniques for the detection and quantification of liver steatosis. Radiographics 29(1):231-260

5. Boyce CJ, Pickhardt PJ, Kim DH, Taylor AJ, Winter TC, Bruce RJ et al (2010) Hepatic steatosis (fatty liver disease) in asymptomatic adults identified by unenhanced low-dose CT. AJR Am J Roentgenol 194(3):623-628

6. Alpern MB, Lawson TL, Foley WD, Perlman SJ, Reif LJ, Arevalos E et al (1986) Focal hepatic masses and fatty infiltration detected by enhanced dynamic CT. Radiology 158(1):45-49

7. Hamer OW, Aguirre DA, Casola G, Lavine JE, Woenckhaus M, Sirlin CB (2006) Fatty liver: imaging patterns and pitfalls. Radiographics 26(6):1637-1653

8. Kim DY, Park SH, Lee SS, Kim HJ, Kim SJ, Kim MY et al (2010) Contrast-enhanced computed tomography for the diagnosis of fatty liver: prospective study with same-day biopsy used as the reference standard. Eur Radiol 20(2):359-366

9. Yokoo T, Bydder M, Hamilton G, Middleton MS, Gamst AC, Wolfson T et al (2009) Nonalcoholic fatty liver disease: diagnostic and fat-grading accuracy of low-flip-angle multiecho gradientrecalled-echo MR imaging at 1.5 T. Radiology 251(1):67-76

10. Oliva MR, Mortele KJ, Segatto E, Glickman JN, Erturk SM, Ros PR, Silverman SG (2006) Computed tomography features of nonalcoholic steatohepatitis with histopathologic correlation. J Comput Assist Tomogr 30(1):37-43

11. Mathieu D, Luciani A, Achab A, Zegai B, Bouanane M, Kobeiter $\mathrm{H}$ (2001) Les pseudolésions hépatiques. Gastroenterol Clin Biol 25:B158-B166

12. Basaran C, Karcaaltincaba M, Akata D, Karabulut N, Akinci D, Ozmen $\mathrm{M}$ et al (2005) Fat-containing lesions of the liver: crosssectional imaging findings with emphasis on MRI. AJR Am J Roentgenol 184(4):1103-1110

13. Matsui O, Kadoya M, Takahashi S, Yoshikawa J, Gabata T, Takashima $\mathrm{T}$ et al (1995) Focal sparing of segment IV in fatty livers shown by sonography and CT: correlation with aberrant gastric venous drainage. AJR Am J Roentgenol 164(5):1137-1140

14. Khalili K, Lan FP, Hanbidge AE, Muradali D, Oreopoulos DG, Wanless IR (2003) Hepatic subcapsular steatosis in response to intraperitoneal insulin delivery: CT findings and prevalence. AJR Am J Roentgenol 180(6):1601-1604

15. Prasad SR, Wang H, Rosas H, Menias CO, Narra VR, Middleton WD et al (2005) Fat-containing lesions of the liver: radiologicpathologic correlation. Radiographics 25(2):321-331 